International Journal of Clinical \& Medical Allergy (IJCMA)

ISSN 2332-2799

\title{
Olive Oil Sensitivity and desensitization: a non-IgE mediated mechanism.
}

Marilyn R. Karam MD', Timothy J. Franxman MD², Marc S. McMorris MD ${ }^{1}$, James L. Baldwin MD ${ }^{1}$

${ }^{1}$ Department of Internal Medicine, Division of Allergy and Clinical Immunology, University of Michigan Health System, Ann Arbor, MI

${ }^{2}$ Allergy and Clinical Immunology, Family Allergy and Asthma, Louisville, KY

\section{Abstract}

Introduction:The pungency of extra virgin olive oil (EVOO) is attributed to one of its phenolic compounds called oleocanthal (OC) and is mediated through the transient receptor channel potential cation channel ankyrin subtype 1 (TRPA1) expressed in the posterior oropharynx. EVOO also mimics the pharmacology of ibuprofen in that both agents cause a dose-dependent inhibition of cyclooxygenase enzymes COX-1 and COX-2. EVOO sensitivity has been described in the literature previously however EVOO desensitization has not. Herein we report the first case of successful EVOO desensitization using a novel EVOO desensitization protocol.

Case description: A 25-year-old patient with medical history significant for aspirin sensitivity, exercise induce asthma and mixed vasomotor and allergic rhinitis presented with a history of facial and tongue pruritus, throat irritation, coughing and hives within minutes of olive oil consumption. Symptoms required diphenhydramine however several prior episodes required epinephrine. A series of food and drug challenges were entertained to establish the mechanism of EVOO sensitivity in this patient. Also a novel EVOO desensitization protocol was devised.

Discussion:EVOO desensitization has not been reported previously in the literature and in this report we describe the first case of successful EVOO desensitization using a novel EVOO desensitization protocol. Furthermore the patient continues to be in a desensitized state 2 years after the procedure maintained with daily doses of $10 \mathrm{ml}$ EVOO. Although the mechanism of her reaction is likely due to OC stimulation of TRPA1, the mechanism of desensitization and maintenance of the desensitized state is unclear and requires further study.

Keywords: Olive Oil; Desensitization; TRPA1.

*Corresponding Author:

Marilyn R. Karam, MD,

Department of Internal Medicine,

Division of Allergy and Clinical Immunology,

University of Michigan Health System,

Ann Arbor, MI,USA

Tel/Fax: 3135108951

E-mail: mkaram@med.umich.edu

Received: July 22, 2014

Accepted: August 18, 2014

Published: August 21, 2014

Citation: Marilyn R. Karam, MD; Timothy J. Franxman, MD; Marc S. McMorris, MD; James L. Baldwin, MD (2014) Olive Oil Sensitivity and desensitization: a non-IgE mediated mechanism 1:201, 8-10. doi: http://dx.doi.org/10.19070/2332-2799-140003

Copyright: Marilyn R. Karam (C) 2014. This is an open-access article distributed under the terms of the Creative Commons Attribution License, which permits unrestricted use, distribution and reproduction in any medium, provided the original author and source are credited.

\section{Chief Complaint}

Olive oil sensitivity

\section{History Of Present Illness}

A 25-year-old nurse presented with a history of facial and tongue pruritus, throat irritation, coughing and hives within minutes of olive oil consumption. Diphenhydramine usually provided reliefhowever several prior episodes required epinephrine. She also related eye and lip pruritus and chest tightness that required epinephrine and diphenhydramine to resolve after inhalation but not consumption of a coworker's olive oil.

\section{Past Medical History (PMH)}

Twenty minutes after taking an aspirin containing product at age 17 , she developed hives, cough and difficulty breathing. She tolerates up to $200 \mathrm{mg}$ of ibuprofen. The patient also has a history of exercise induced asthma which is well controlled on as needed albuterol inhaler and mixed (vasomotor and allergic) rhinitis with sensitivities to grass and mold also well controlled on nasal corticosteroids. She has intermittent gastroesophagealreflux controlled on as needed proton pump inhibitors and essential tremors on 10 mg of propranolol that she takes on her work days, but not on other days. There is no latex sensitivity history. 


\section{Social And Environmental History}

She is a married pediatric ER nurse with 2 dogs and no smokers in her environment. She does not smoke, drink or use illicit drugs.

\section{Family History}

Positive for food allergies (seafood in her mother and peanut in her nephew), insect sting reactivity, cerebrovascular disease, coronary artery disease and thyroid dysfunction.

\section{Physical Examination}

The patient is a well-developed, well-nourished young woman, in no acute distress, with the following vitals: blood pressure 100/70, pulse 73 , respiratory rate of 14 and afebrile. Head, eyes, ears, nose and throat examination was within normal limits with no nasal polyps. Chest exam revealed good bilateral breath sounds with no wheezing, crackles or rhonchi. Cardiac rhythm was regular S1 S2 with no murmurs. Abdomen was soft, non-tender without hepatosplenomegaly or masses. Extremities showed no clubbing, cyanosis or edema and her skin was clear without lesions.

\section{Initial Laboratory Findings}

Skin prick testing for black olive oil, green olive oil, Da Vinci ${ }$ extra virgin olive oil (EVOO), and Berio ${ }^{\circledR}$ olive oil, were all negative with appropriate negative saline control and histamine control (4 $\mathrm{mm}$ wheal and $10 \mathrm{~mm}$ flare). Olive tree pollen and olive food specific $\operatorname{IgE}$ levels were negative $(<3.5 \mathrm{kU} / \mathrm{L})$ with a total $\operatorname{IgE}$ level of $16 \mathrm{kU} / \mathrm{L}$.

\section{Futher Diagnostic Studies and Clinical Course}

Since clinical symptoms were suggestive of an IgE-mediated food allergy despite negative skin and ImmunoCAP tests, inhalational and oral placebo controlled food challenges were done.

\section{EVOO inhalation challenge}

Two different forms of home-made raspberry salad dressing were used for the inhalation challenge each containing either EVOO or canola oil mixed with raspberry vinegar and raspberry jam. The patient successfully passed the inhalational challenge.

\section{EVOO oral challenge}

Home-made raspberry salad dressings containing mixtures of EVOO and canola oil mixed with raspberry vinegar and raspberry jam were used with gradually increasing EVOO content. Within minutes of ingesting a $60 \%$ EVOO oil mixture, shortness of breath, eye and throat pruritus with a 7\% drop inFEV1 was noted. Symptoms resolved over the course of 45 minutes after administration of $25 \mathrm{mg}$ diphenhydramine.

\section{Pathophysiologic Mechanism}

The pungency of EVOO is attributed to one of its phenolic compounds called oleocanthal (OC) and is mediated through the transient receptor channel potential cation channel ankyrin subtype 1 (TRPA1) expressed in the posterior oropharynx[1,8,9]. EVOO also mimics the pharmacology of ibuprofen in that both agents cause a dose-dependent inhibition of cyclooxygenase enzymes COX-1 and COX-2[2]. Additionally ibuprofen (but not aspirin) also stimulates the same TRPA1 receptors present on the trigeminal nervous system that innervates taste buds in the posterior oropharynx [3]. Therefore two possible non- $\mathrm{IgE}$ mediated mechanisms for EVOO sensitivity in this patient were entertained: 1) inhibition of COX pathway or 2) TRPA1stimulation.

Given, the patient's history of aspirin (ASA) sensitivity, the COX inhibition mechanism was further explored with a series of challenges.

\section{Open ASA challenge}

Twenty three minutes after ingesting $81 \mathrm{mg}$ of ASA unblinded, hives on her left lower jaw and left knee and mild itching of the posterior oropharynx ensued. Vital signs, oxygen saturation and FEV1remained stable. Symptoms persisted for an additional 30 minutes and resolved with $10 \mathrm{mg}$ cetirizine over 45 minutes.

\section{Double blind placebo controlled ASA challenge}

Thirty five minutes after $650 \mathrm{mg}$ ASA (not placebo), throat pruritus and hives with stable vital signs and FEV1 were noted and again responded to $10 \mathrm{mg}$ cetirizine. A tryptase level was drawn and was normal $(4.2 \mathrm{ng} / \mathrm{mL})$.

\section{Open oral liquid ibuprofen challenge}

$400 \mathrm{mg}$ of oral liquid ibuprofen elicited no signs or symptoms over 3 hours of monitoring Vital signs and FEV1 remained stable.

\section{Open oral aminosaicylic acid challenge}

A $400 \mathrm{mg}$ oral 5-aminosalicylic acid challenge was negative.

\section{Interpretation of results of challenges}

Given the time course and positive results of the open and blinded ASA challenges and the negative results of the ibuprofen and aminosalicylic acid it is unlikely that her ASA symptoms were a result of either COX-1 inhibition mechanism or salicylate sensitivity, rather this likely represents a unique "single agent" NSAID (ASA) sensitivity and possibly an IgE-mediated type. The time course supports this mechanism as well, though tryptase was negative and skin testing to ASA was not done. Furthermore, it is unlikely that the patient's ASA sensitivity has any relation to her EVOO sensitivity based on the known physiology of EVOO pungency. Rather, we propose two unrelated mechanisms for the EVOO and ASA reactions.

Although successful desensitization of aspirin and NSAIDs is well documented, desensitization to EVOO has not been described. However, at the patient's request we devised a 3 daygraded dose desensitization protocol with EVOO in order to reach tolerance of a dose of at least $10 \mathrm{ml}$.

\section{EVOO Desensitization Protocol}

Day 1 week 1: Every 30 minute oral doses of 10\% EVOO in canola oil: $1 \mathrm{ml}, 2 \mathrm{ml}, 4 \mathrm{ml}$ and $8 \mathrm{ml}$ elicited no symptoms.

Between week 1 and 2 the patient consumed $0.8 \mathrm{ml}$ of $100 \%$ 
EVOO twice daily.

Day 2 Week 2: Every 30 minutes oral $2 \mathrm{ml}, 4 \mathrm{ml}$ and $6 \mathrm{ml}$ quantities of $50 \%$ EVOO in canola oil were dosed. At $6 \mathrm{ml}$, chest tightness, cough, eye and throat pruritus ensued within minutes without change in FEV1 and resolvedcompletely an hour after receiving an albuterol nebulizer treatment, $50 \mathrm{mg}$ of diphenhydramine and $10 \mathrm{mg}$ of montelukast.

Between week 2 and 3 the patient consumed $4 \mathrm{ml}$ of $50 \%$ EVOO twice daily.

Day 3 Week 3: Q 30 minute oral doses of 100\% EVOO: 2ml, $3 \mathrm{ml}, 4 \mathrm{ml}$ and $5 \mathrm{ml}$ elicited no symptoms. The patient was instructed to consume $10 \mathrm{ml}$ daily EVOO to maintain the desensitized state. Two years later she continues to tolerate $10 \mathrm{ml}$ of EVOO daily.

\section{Discussion}

EVOO is composed of phenolic compounds of the secoiridoidfamily. Its pungency is attributed to one of its phenolic compounds, the dialdehyde form of deacetoxy-ligstrosideaglycone also known as oleocanthal (oleo- for olive, canth- for sting and alfor aldehyde)[8,9].OC irritation is localized to theoropharyngeal region of the oral cavity and is highly variable among individuals. In contrast to most irritants, OC does not elicit generalized irritation of mucous membranes throughout the oral cavity[1]. Instead it has been demonstrated that both $\mathrm{OC}$ and ibuprofen, activate the TRPA1 ex vivo, and excite the trigeminal nervous system that innervates taste buds. Immunohistochemicalimaging of human tissues shows that neural fibers of the sensitive pharyngeal and nasal epithelia have a higher content of TRPA1 compared to anterior tongue. To date, TRPA1 channels have not been reported on neuronal fibers innervating the human anterior tongue likely explaining the localization of OC irritation to the posterior oropharynx.Additionally, both the vanilloid 1 (TRPV1) and TRPA1 have been identified as sensors of airway irritation and initiator of the cough reflex[5]. It has been postulated thatbronchial epithelial TRPV1 stimulation by environmental irritants might sensitize airway sensory neurons and contribute to the airway and cough hypersensitivity that characterizes asthma. Indeed, significantly more TRPV1 channels were found to be present in the human airway epithelium of patients with refractory asthma compared to non-asthmatic patients6. More recently, TRPV1 and TRPA1 positive neurons have been shown to have an IL-31 receptor which is a functional neural cytokine receptor involved in acute and chronic itch[4].

EVOO desensitization has not been previously described in the literature. Herein we report thefirst case of successful EVOO desensitization using a novel EVOO desensitization protocol. Furthermore the patient continues to be in a desensitized state 2 years after the procedure maintained with daily doses of $10 \mathrm{ml}$ EVOO. Although the mechanism of her reaction is likely due to OC stimulation of TRPA1, the mechanism of desensitization and maintenance of the desensitized state is unclear and requires further study.

\section{References}

[1]. Cicerale S Breslin P Beauchamp GK Keast RS. (2009) Sensory Characterization of the Irritant Properties ofOleocanthal, a Natural Anti-inflammatory Agent in Extra Virgin Olive Oil. Chem. Senses. 34(4): 333-339.

[2]. Beauchamp GK Keast RS, Morel D (2005)Phytochemistry: Ibuprofen-like activity in extra-virgin olive oil.Nature; 437(7055):45-46

[3]. Peyrot des Gachons C Uchida K Bryant B (2011)Unusual pungency from extra-virgin olive oil is attributable to restricted spatial expression of the receptor of oleocanthal. JNeurosci; 31(3):999-1009

[4]. Cevikbas F Wang X Akiyama T (2014) A sensory neuron-expressed IL-31 receptor mediates T helper cell-dependent itch: involvement of TRPV1 and TRPA1. J Allergy Clin Immunol;133(2):448-460.

[5]. Geppeetti P Patacchini R Nassini R (2010) Cough: the emerging role of the TRPA1 channel. Lung; 188(Suppl1):S63-S68.

[6]. McGravey L Butler C Stokesberry S (2014) Increased expression of bronchial epithelial transient receptor potentialvanilloid 1 channels in patients with severe asthma. J AllergyClin Immunol; 133:704-12.

[7]. Birrell M Bonvini S Dubuis E (2014) Tiotropiummodulates transient receptor potential V1 (TRPV1) in airways sensory nerves: A beneficial offtarget effect?. J Allergy ClinImmunol; 133:679-87.

[8]. Franxman T Baldwin JL (2014) Pharmacologic food reactions. In: Metcalfe D, Ed., Food Allergy: Adverse Reactions to Foods and Food Additives, John Wiley \& Sons, 43-451.

[9]. Fogliano V Sacchi R (2006) Oleocanthal in olive oil: between myth and reality. Mol Nutr Food Res; 50(1):5-6. 